\title{
Assessing kinesthetic proprioceptive function of the upper limb: A novel dynamic movement reproduction task using a robotic arm
}

\author{
Kristof Vandael $^{1,2}$, Tasha R Stanton ${ }^{3,4}$, Ann Meulders ${ }^{\text {Corresp. 1,5 }}$ \\ ${ }^{1}$ Experimental Health Psychology, University of Maastricht, Maastricht, Netherlands \\ 2 Laboratory of Biological Psychology, Katholieke Universiteit Leuven, Leuven, Belgium \\ 3 Neuroscience Research Australia, Randwick, New South Wales, Australia \\ 4 IIMPACT in Health, University of South Australia, Adelaide, South Australia, Australia \\ 5 Research Group Health Psychology, Katholieke Universiteit Leuven, Leuven, Belgium \\ Corresponding Author: Ann Meulders \\ Email address: ann.meulders@kuleuven.be
}

Background. Proprioception refers to the perception of motion and position of the body or body segments in space. A wide range of proprioceptive tests exists, although tests dynamically evaluating sensorimotor integration during upper limb movement are scarce. We introduce a novel task to evaluate kinesthetic proprioceptive function during complex upper limb movements using a robotic device. We aimed to evaluate the test-retest reliability of this newly developed Dynamic Movement Reproduction (DMR) task. Furthermore, we assessed reliability of the commonly used Joint Reposition (JR) task of the elbow, evaluated the association between both tasks, and explored the influence of visual information (viewing arm movement or not) on performance during both tasks.

Methods. During the DMR task, participants actively reproduced movement patterns while holding a handle attached to the robotic arm, with the device encoding actual position throughout movement. In the JR task, participants actively reproduced forearm positions; with the final arm position evaluated using an angle measurement tool. The difference between target movement pattern/position and reproduced movement pattern/position served as measures of accuracy. In study $1(\mathrm{~N}=23)$, pain-free participants performed both tasks at two test sessions, 24-hours apart, both with and without visual information available (i.e., vision occluded using a blindfold). In study $2(\mathrm{~N}=64)$, an independent sample of pain-free participants performed the same tasks in a single session to replicate findings regarding the association between both tasks and the influence of visual information.

Results. The DMR task accuracy showed good-to-excellent test-retest reliability, while JR task reliability was poor: measurements did not remain sufficiently stable over testing days. The DMR and JR tasks were only weakly associated. Adding visual information (i.e., watching arm movement) had different performance effects on the tasks: it increased JR accuracy but decreased DMR accuracy, though only when the DMR task started with visual information available (i.e., an order effect).

Discussion. The DMR task's highly standardized protocol (i.e., largely automated), precise measurement and involvement of the entire upper limb kinetic chain (i.e., shoulder, elbow and wrist joints) make it a promising tool. Moreover, the poor association between the JR and DMR tasks indicates that they likely capture unique aspects of proprioceptive function. While the former mainly captures position sense, the latter appears to capture sensorimotor integration processes underlying kinesthesia, largely independent of position sense. Finally our results show that the integration of visual and proprioceptive information is Peer) reviewing PDF | (2020:11:55170:1:0:NEW 19 Feb 2021) 
not straightforward: additional visual information of arm movement does not necessarily make active movement reproduction more accurate, on the contrary, when movement is complex, vision appears to make it worse. 
2 Assessing kinesthetic proprioceptive function of the

3 upper limb: A novel dynamic movement reproduction

4 task using a robotic arm

7

8 Kristof Vandael ${ }^{1,2}$, Tasha R. Stanton ${ }^{3,4}$, \& Ann Meulders ${ }^{1,5^{*}}$

$9 \quad{ }^{1}$ Experimental Health Psychology, Maastricht University, Maastricht, The Netherlands

$10{ }^{2}$ Laboratory of Biological Psychology, KU Leuven, Leuven, Belgium

$11{ }^{3}$ IIMPACT in Health, University of South Australia, Adelaide, South Australia, Australia

$12{ }^{4}$ Neuroscience Research Australia, Randwick, New South Wales, Australia

${ }^{5}$ Research Group Health Psychology, KU Leuven, Leuven, Belgium

$15 *$ Corresponding Author:

16 Ann Meulders

17 Universiteitssingel 40, Maastricht, 6229 ER, The Netherlands

18 Email address: ann.meulders@kuleuven.be 
19

20

21

22

23

24

25

26

27

28

29

30

31

32

33

34

35

36

37

38

39

40

41

42

43

44

45

46

47

48

49

50

51

52

53

\section{Abstract}

Background. Proprioception refers to the perception of motion and position of the body or body segments in space. A wide range of proprioceptive tests exists, although tests dynamically evaluating sensorimotor integration during upper limb movement are scarce. We introduce a novel task to evaluate kinesthetic proprioceptive function during complex upper limb movements using a robotic device. We aimed to evaluate the test-retest reliability of this newly developed Dynamic Movement Reproduction (DMR) task. Furthermore, we assessed reliability of the commonly used Joint Reposition (JR) task of the elbow, evaluated the association between both tasks, and explored the influence of visual information (viewing arm movement or not) on performance during both tasks.

Methods. During the DMR task, participants actively reproduced movement patterns while holding a handle attached to the robotic arm, with the device encoding actual position throughout movement. In the JR task, participants actively reproduced forearm positions; with the final arm position evaluated using an angle measurement tool. The difference between target movement pattern/position and reproduced movement pattern/position served as measures of accuracy. In study $1(\mathrm{~N}=23)$, pain-free participants performed both tasks at two test sessions, 24-hours apart, both with and without visual information available (i.e., vision occluded using a blindfold). In study $2(\mathrm{~N}=64)$, an independent sample of pain-free participants performed the same tasks in a single session to replicate findings regarding the association between both tasks and the influence of visual information.

Results. The DMR task accuracy showed good-to-excellent test-retest reliability, while JR task reliability was poor: measurements did not remain sufficiently stable over testing days. The DMR and JR tasks were only weakly associated. Adding visual information (i.e., watching arm movement) had different performance effects on the tasks: it increased JR accuracy but decreased DMR accuracy, though only when the DMR task started with visual information available (i.e., an order effect).

Discussion. The DMR task's highly standardized protocol (i.e., largely automated), precise measurement and involvement of the entire upper limb kinetic chain (i.e., shoulder, elbow and wrist joints) make it a promising tool. Moreover, the poor association between the JR and DMR tasks indicates that they likely capture unique aspects of proprioceptive function. While the former mainly captures position sense, the latter appears to capture sensorimotor integration processes underlying kinesthesia, largely independent of position sense. Finally, our results show that the integration of visual and proprioceptive information is not straightforward: additional visual information of arm movement does not necessarily make active movement reproduction more accurate, on the contrary, when movement is complex, vision appears to make it worse. 


\section{Introduction}

55

56

57

58

59

60

61

62

63

64

65

66

67

68

69

70

71

72

73

74

75

76

77

78

79

80

81

82

83

84

85

86

87

88

89

90

91

92

93

When we perform controlled voluntary movements, such as reaching for a glass of water, we rely heavily upon sensory information elicited from the movement to successfully perform and control that movement. A key source of sensory information is proprioceptive input - it allows for the perception of motion and position of the body or body segments in space (Proske \& Gandevia, 2012). Proprioceptive input consists of an ensemble of sensory information from various receptors that detect and encode the mechanical changes in tissues (e.g., muscles, skin) during movement. During active movement, muscle spindles are considered the primary source of proprioceptive information (Proske \& Gandevia, 2012). Proprioceptive input then undergoes processing within the spinal cord, cephalad transmission up the sensory neuraxis, finally leading to a proprioceptive representation within the brain (i.e., area 2 of the primary somatosensory cortex in case of arm movement; Chowdhury, Glaser, \& Miller, 2020). During movement, proprioceptive (and tactile) input is used to inform motor planning (Wolpert, Goodbody, \& Husain, 1998). It is also used to determine whether or not the movement has occurred as intended - i.e., a motor efferent copy is generated and compared to the sensory input that has resulted as a consequence of this movement (Wolpert \& Ghahramani, 2000; Wolpert et al., 1995). Such a process of sensorimotor integration ultimately allows for accurate, controlled movement.

A large variety of tests exist to quantify proprioceptive function, which differ in the required motor and memory capacity to perform the test, but importantly, also vary in the aspect of proprioception that they evaluate (Hillier, Immink, \& Thewlis, 2015). One aspect of proprioceptive function involves the perception of motion (i.e., kinesthesia), which is typically evaluated using a task in which the joint of interest is passively moved until the subject indicates they sense the movement and/or its direction (Juul-Kristensen et al., 2008b). Alternatively, to assess perception of spatial location or position (i.e., position sense), limb position reproduction tasks, such as the Joint Repositioning (JR) task are commonly used (Han, Waddington, Adams, Anson, \& Liu, 2016). In the active variant, participants have their vision occluded and reproduce target positions using the body part of interest (e.g., using target positions of the forearm to assess position sense at the elbow joint). The average difference between target and reproduced position then serves as a measure of accuracy.

A limitation of these proprioceptive tasks is that they generally do not allow for evaluation of more complex processes that are essential for accurate and controlled movement (i.e., kinesthesia during functional movement), such as integration of sensory and motor information. This is an important limitation because goal-directed movement requires dynamic updating of motor output based on proprioceptively encoded (and changing) body position (i.e., sensorimotor integration; Proske \& Gandevia, 2012). Evaluation of such processes underlying kinesthesia during active movement may provide unique and important information, given that there are known dynamic modulations that occur during movement (e.g., sensory gating; Saradjian, 2015). Capturing processes of dynamic modulation may also be important because proprioceptive tasks evaluating position sense or passively evaluating kinesthesia provide little 
94 insight into dynamic movement; that is, they are not always associated with actual motor 95 performance (Davies, Krauschaar, Brinks, \& Jennings, 2006; Dukelow, Herter, Bagg, \& Scott, 96 2012; Helsen et al., 2016; Kitchen \& Miall, 2019). Here we introduce a novel task in which 97 movement patterns are reproduced to dynamically assess kinesthetic proprioceptive function: 98 the Dynamic Movement Reproduction (DMR) task. Using the HapticMaster (Motekforce Link, 99 Amsterdam, Netherlands), a 3 degrees-of-freedom, force-controlled robotic arm, this task 100 involves continuous (i.e., online) assessment of an actively reproduced arm movement, thus

101

102

103

104

105

106

107

108

109

110

111

112

113

114

115

116

117

118

119

120

121

122

123

124

125

126

127

128

129

130

131

132 including aspects of both limb position sense and sensorimotor integration to support kinesthetic function. The ability to accurately assess kinesthetic proprioceptive function during complex movement processes is clinically relevant given that a wide range of clinical conditions are characterized by impaired proprioception (Goble, 2010; Proske \& Gandevia, 2012; Röijezon, Clark, \& Treleaven, 2015) and that the type of proprioceptive deficit can vary (Kenzie, Semrau, Hill, Scott, \& Dukelow, 2017), meaning that it may be integration processes (versus position sense) that are of crucial importance in certain clinical conditions.

A key feature for both research and clinical relevance of a proprioceptive function task is adequate test-retest reliability. Past work shows that the reliability of traditional, active JR tests ranges widely depending on the device used and the extremity joint measured (Clark, Röijezon, \& Treleaven, 2015; Elangovan, Herrmann, \& Konczak, 2014). Equipment measurement error likely influences these reliability findings. Use of more sophisticated equipment during testing, such as robotic devices (e.g. the HapticMaster) - which are becoming increasingly prevalent in research and clinical practice - may have higher sensitivity and precision (Maggioni et al., 2016). Such properties also affect the ability to detect proprioceptive impairment, which is essential given that even slight impairments might be of clinical relevance, particularly for complex sensorimotor integration processes. Therefore, the primary aims of the current study were to evaluate (1) test-retest reliability of the DMR task and (2) a JR test of the elbow, and (3) the association between performance on both tasks. Understanding the association between the tasks is important - if highly associated, then a complex task (such as the DMR task) might not be needed; if only weakly associated, then it would provide evidence that these tasks capture different aspects of proprioceptive function. Thus to evaluate these aims, in Study 1, healthy participants performed the DMR and JR tasks at two different test sessions, 24-hours apart. Since the use of a robotic device allows for a highly standardized protocol and precise measurement, i.e., features shown to increase test-retest reliability (Maggioni et al., 2016), we hypothesised that (1) the DMR task would be highly reliable (good-to-excellent range). Furthermore, consistent with findings from Juul-Kristensen and colleagues (2008b), we hypothesised that (2) JR accuracy at the elbow would have fair-to-good reliability. Finally, we predicted (3) a weak association between DMR and JR accuracy. Both tasks involve active elbow movements and involve aspects of joint position sense; however, the continuous measurement of error during the DMR task likely also captures complex sensorimotor integration processes, thus only a weak association was anticipated. 
133

134

135

136

137

138

139

140

141

142

143

144

145

146

147

148

149

150

151

152

153

154

155

156

157

158

159

160

161

162

163

164

165

166

167

168

169

170

171

172

In addition, to better understand the various sensory contributions to task performance (i.e., a task involving sensorimotor integration), our secondary aim was to evaluate the influence of visual information on both proprioceptive measures. Movements typically involve integration of visual and proprioceptive information, which may be combined in differing ways based on the nature of movement (e.g., differing between trajectory control and final position regulation; Scheidt, Conditt, Secco, \& Mussa-Ivaldi, 2005). Testing both tasks with and without visual information of limb movement allows us to determine the relative visual versus proprioceptive weighting in task performance. We hypothesised that there would be increased accuracy with visual information for both tasks, given that vision provides an extra source of sensory information that may assist in movement and joint position accuracy. Given that recent calls to improve research rigor recommend undertaking validation of study findings in an independent sample (Laraway, Snycerski, Pradhan, \& Huitema, 2019), we also evaluated the two proprioceptive tasks (with and without visual information available) in a second independent sample (Study 2) to ensure reproducibility of our findings.

\section{Study 1: Materials \& Methods}

\section{Participants}

Twenty-nine pain-free volunteers [sample size based on Juul-Kristensen et al. (2008b)] were recruited through word-of-mouth and the participant recruitment system of Maastricht University (Sona; Sona Systems, Nijmegen, The Netherlands). Six participants were excluded: four due to equipment failure and two because they confused movement directions (e.g., performing clockwise movements, when counterclockwise movements were requested). Statistical analyses were run on the final sample of $N=23$ (mean $(S D)$ age $=24.39$ (3.12), ranging from 18-32, 11 women). Exclusion criteria were: chronic pain; left-handedness; uncorrected problems with hearing or vision; current pain at the dominant hand, wrist, elbow, or shoulder that may hinder task performance. Participants received $€ 7.5$ in gift vouchers as compensation for their time and effort.

\section{Pre-registration of the protocol and ethical approval}

The experimental protocol was approved by the Ethics Review Committee Psychology and Neuroscience of Maastricht University (185 09112017 S5) and pre-registered on AsPredicted (https://aspredicted.org/blind.php? $\mathrm{x}=\mathrm{c} 7 \mathrm{~g} 8 \mathrm{bp})$. Prior to the start of the experiment, all participants read an information sheet, completed an exclusion criteria checklist, and provided written informed consent.

\section{Study design}

All participants performed both the JR and the DMR tasks using the dominant (right) arm. Each task comprised two conditions: Visual Information (i.e., without blindfold) and No Visual Information (i.e., with blindfold). In the Visual Information condition, participants 
173 directly watched the movement of their own limb. The order of the tasks and conditions was

174 randomized across participants (using random.org). The same tasks were performed 24 hours

175 later, in the same order as during the first test session (Figure 1).

176

177

Apparatus

178

179

180

181

182

183

184

185

186

187

188

189

190

191

192

193

194

195

196

197

198

199

200

201

202

203

204

205

206

207

208

209

210

Angle measurement tool (JR task).

The Bosch GLM 80 Professional measuring tool (Robert Bosch GmbH, GerlingenSchillerhoehe, Germany) was used to measure arm positions (in degrees; precision $=0.1^{\circ}$; accuracy $= \pm 0.2^{\circ}$ ). The device was attached to participants' wrist using a Velcro strap and measured the angle of the forearm relative to the horizontal surface.

\section{HapticMaster (DMR task).}

The HapticMaster (Motekforce Link, Amsterdam, the Netherlands; Figure 2) is a 3 degrees-of-freedom force-controlled robotic arm. Participants hold the handle of the device and can move it in all directions within a specific volume of space. The HapticMaster allows forward/backward movement with a depth of $40 \mathrm{~cm}$, upward/downward movement with a height of $40 \mathrm{~cm}$, and 60 degrees of rotation around its vertical axis (with smallest radius $46 \mathrm{~cm}$; see youtube.com/watch? $=\mathrm{AQNNaEZ}$ KIlg for a demonstration). In the current task, movements were confined to a 2-dimensional horizontal movement plane (i.e., height remained constant). The robotic arm can be moved by exerting force on a sensor (i.e. circular handgrip) attached at the end of the arm. The HapticMaster automatically logs position along all three dimensions every $2 \mathrm{~ms}$, with a resolution of $10^{\wedge}-6 \mathrm{~m}$.

\section{Computer software and hardware (DMR task).}

The DMR task was programmed in C\# using Unity 2017 (Unity Technologies, San Francisco, CA, USA). The experimental task was run on a Windows 10 Enterprise (Microsoft Corporation, Redmond, WA, USA) 64-bit Intel Core desktop computer (Intel Corporation, Santa Clara, CA, USA) and instructions were presented on a 40-inch LCD screen (Samsung UE40ES5500; Samsung Group, Seoul, South Korea). A Windows 10 compatible foot switch (USB Triple Foot Switch II; Scythe Co., Ltd., Tokyo, Japan) was used to navigate through instructions.

\section{Experimental setting}

In both tasks, participants sat with their back against the chair and a strap encircling their torso to ensure their position remained fixed. For the DMR task, participants sat in front of the HapticMaster within reaching distance of the sensor (Figure 2). The LCD screen was mounted on the wall in front of them; the foot switch was placed on the floor at their feet. The experimenter sat on the opposite side of a partition and observed participants via a webcam. For the JR task, participants sat with their right elbow resting on a marked position on a desk. During this task, the experimenter sat next to them to read the angles of the forearm positions.

211

212 Procedure 
213

214

215

216

217

218

219

220

221

222

223

224

225

226

227

228

229

230

231

232

233

234

235

236

237

238

239

240

241

242

243

244

245

246

247

248

249

250

251

252

\section{Dynamic movement reproduction task.}

During the DMR task, participants replicated square (practice) and circular movement patterns while holding the sensor of the HapticMaster. Movement direction was counterbalanced: some participants moved in the clockwise direction during the entire Visual Information condition and counterclockwise during the entire No Visual Information condition (Figure 1), other participants received the reversed combinations. Therefore, none of the findings can be attributed to specific combinations of stimuli. Each condition started with three practice trials to familiarize participants with the procedure, followed by six test trials.

Practice phase. Instructions on how to operate the HapticMaster and the procedure of the task were presented on-screen, including movement direction (and pattern shape; Figure 1), and whether or not a blindfold would be worn. Additionally, participants were informed that all movements would occur in the horizontal plane. On each trial, the HapticMaster first restricted movement to a single trajectory to show participants what movement was to be reproduced (i.e., target movement). The participants actively explored the trajectory; the HapticMaster was programmed to haptically block certain areas of its workspace, thus restricting the movement to specific patterns. During practice, this pattern was a square with a side length of $16 \mathrm{~cm}$. Note that on all practice trials the shape of this pattern was the same and starting positions were always in the middle of the side closest to the participant. Participants began movement when they heard a starting tone after the automated audio message "Start guided movement" (Figure 2). If participants moved in the wrong direction, an error message was played and the trial restarted. After being guided through the target movement once, they were asked to reproduce this movement as accurately as possible, while having the entire range of the robotic arm - within the horizontal plane - available. Participants began moving when they heard a starting tone after the automated audio message "Start free movement", and participants verbally said, "Stop" when they finished reproducing the movement, at which time the experimenter manually ended the trial. The robotic arm then moved to a new starting position for the next trial. Six different starting positions within the same horizontal plane were used (random order; shoulder angles along the frontal plane approximately between 0 and 90 degrees; shoulder angles along the longitudinal plane approximately between 0 and 45 degrees; elbow angle approximately between 30 and 160 degrees) to limit potential spatial learning effects. No feedback regarding participants' performance was given.

Test phase. Identical procedures to the practice phase were used except the shape of the target movements was changed to a circle with a radius of $8 \mathrm{~cm}$ (to limit potential training effects). Note that on all test trials the shape of this pattern was the same and the starting position was always on the point of the circular pattern closest to the participant.

\section{Joint reposition task.}

Each condition started with one practice trial to familiarize participants with the procedure, followed by six test trials. During the task, participants' elbow rested on a marked spot on a horizontal surface (shoulder angle along the frontal plane at approximately 0 degrees; elbow angle at approximately 135 degrees; wrist in neutral position, with thumb pointing 
253

254

255

256

257

258

259

260

261

262

263

264

265

266

267

268

269

270

271

272

273

274

275

276

277

278

279

280

281

282

283

284

285

286

287

288

289

290

291

292

upwards; see Figure 1). Prompted verbally by the experimenter, participants actively moved their forearm to a target position, moved back to resting position on the horizontal surface, and then actively reproduced the target position (Figure 3). Participants were allowed to adjust the position of the forearm until they felt it reached the correct position, and verbally indicated when this was the case. Both the target and reproduced angle were recorded by the experimenter. Three different target angles $\left(30^{\circ}, 45^{\circ}\right.$ and $60^{\circ}$; between forearm and horizontal surface) presented in random order were assessed twice. No feedback regarding participants' performance was given.

\section{Main outcome variables}

\section{Dynamic movement reproduction error.}

DMR error was operationalized as the mean absolute difference (in $\mathrm{cm}$ ) between the reproduced and the target circular movement pattern (i.e., radius) on each trial (see Supplementary Material S1 for an alternative measure considering direction of errors). Larger errors reflect poorer accuracy. The reproduced radius was calculated using the coordinates of each performed movement, as logged by the HapticMaster (Figure 4).

\section{Joint reposition error.}

JR error is operationalized as the absolute difference between the target angle and the reproduced angle on each trial. Again, larger errors reflect poorer accuracy. The recorded target angles were approximately 30,45 or 60 degrees - in most participants the recorded target angles were not exactly these values due to delay in stopping movement.

\section{Data preparation and statistical analysis overview}

First, data were checked to determine if participants moved in the correct direction during the DMR task. This was assessed using the number of mistakes during guided movements, which was automatically logged by the HapticMaster. Next, data were visually inspected for other artifacts such as participants reaching the end of the movement plane of the HapticMaster.

The mean of each outcome variable was calculated per condition (over 6 trials) and is referred to as accuracy. The standard deviation of the measurements per condition is referred to as consistency, as it indicates whether subjects are consistent in their size/range of error (JuulKristensen et al., 2008b). Bland Altmann plots were used to visually inspect test-retest data of both tasks. Paired t-tests and Repeated Measures Analyses of Variance (RM ANOVAs) were calculated to check for systematic differences between sessions and trials. Intraclass Correlation Coefficients (ICCs; two-way mixed; McGraw \& Wong, 1996; Shrout \& Fleiss, 1979) were used to test the absolute agreement between the test and retest sessions for accuracy and consistency (average measures). The categories of reliability used for reference were .00-.40 (poor), .40-.75 (fair-to-good), and .75-1.00 (good-to-excellent; Fleiss, 1986). We quantified measurement error of the DMR accuracy measure with the Smallest Real Difference (SRD; Beckerman et al., 2001). The SRD of a test is useful for both researchers and clinicians to determine whether a change in accuracy on the individual level is of significance at the $95 \%$ confidence level. First, the 
293 Standard Error of Measurement (SEM) was calculated using the standard deviation (SD) of all

294 test-retest scores and the ICCs: SEM $=$ SD $\times \sqrt{ } 1$-ICC (Chen, Chen, Hsueh, Huang, \& Hsieh, 295 2009). Next, the SEM was used to calculate the SRD: $S R D=1.96 \times \mathrm{SEM} \times \sqrt{2}$. To evaluate if 296 associations existed between task performance on both tasks, Spearman rank-order correlations, $297 \rho$, were calculated between DMR and JR accuracy, and consistency (DMR accuracy in Session 2, 298 and JR accuracy and consistency in Session 1 were not normally distributed; Shapiro Wilk test $299 p<.05)$. All correlations were calculated with and without outliers $(>+3 S D$ or $<-3 S D)$. All 300 analyses were performed on data from the no visual information condition, as this is the

301

302

303

304

305

306

307

308

309

310

311

312

313

314

315

316

317

318

319

320

321

322

323

324

325

326

327

328

329

330

331

332

preferred way to test proprioceptive function. To test the influence of visual information on task performance, RM ANOVAs were conducted on DMR and JR accuracy. The family-wise $\alpha$ was kept at .05. Bonferroni corrections were used to account for multiple testing. All statistical analyses were performed using SPSS 25 (IBM, Armonk, NY, USA). HapticMaster data was preprocessed using a MATLAB script (The MathWorks Inc., Natick, MA, USA).

\section{Study 1: Results}

\section{Test-retest reliability}

Dynamic movement reproduction accuracy (no visual information).

Bland Altmann plots (Figure 5) suggest that there is sufficient test-retest reliability for DMR accuracy and consistency. There was no statistically significant difference between sessions for accuracy, $t(22)=1.54, p=.14$, nor consistency, $t(22)=-0.12, p=.90$. Adding trial as a factor in a RM ANOVA on DMR accuracy indicated no systematic differences on this level $(F<1)$. DMR accuracy had good-to-excellent reliability, $I C C=.80, F(22,22)=5.35, p<.001$, $95 \% C I=[.55-.92]$. The SRD value for DMR accuracy is $.76 \mathrm{~cm}$. In other words, a change between two measurements of the same subject exceeding $.76 \mathrm{~cm}$ can be interpreted as a true change at the $95 \%$ confidence level. Consistency showed fair-to-good reliability, ICC $=.63$, $F(22,22)=2.64, p=.01,95 \% C I=[.11-.85]$. Sensitivity analyses without outliers yielded similar results.

\section{Joint reposition accuracy (no visual information).}

Bland Altmann plots (Figure 6) suggest poor test-retest reliability for JR accuracy and consistency, as variation in means of sessions (i.e., between-subjects) was lower than variation in differences between sessions (i.e., within-subjects). No systematic differences between sessions were present; Accuracy: $t(22)=-0.61, p=.55$; Consistency: $t(22)=-0.01, p=.99$. Adding trial as a factor in a RM ANOVA on JR accuracy indicated no systematic differences on this level, $F(5,110)=1.06, p=.40$. JR accuracy had fair-to-good reliability, $I C C=.46, F(22,22)=1.83, p=.08$, $95 \% C I=[-.29-.77]$, but this was not statistically significant (ICC did not significantly differ from zero). Analysis without outliers confirmed the poor reliability: the ICC dropped to $-.76(F<1)$. JR consistency had poor reliability, $I C C=.33, F(22,22)=1.46, p=.19,95 \% C I=[-.66-.72]$ and was not statistically significant. Analysis without outliers confirmed the poor reliability for consistency, $I C C=-.26, F<1$. 
333

334

335

336

337

338

339

340

341

342

343

344

345

346

347

348

349

350

351

352

353

354

355

356

357

358

359

360

361

362

363

364

365

366

367

368

369

370

371

372

Association between performance accuracy on the dynamic movement reproduction and the joint reposition tasks (no visual information)

The Spearman correlations showed no relationship between DMR and JR accuracy during initial test sessions (Table 1). The retest sessions did show a significant positive correlation of moderate strength. This correlation remained significant when correcting for multiple testing, though no longer when conducting the analyses without outliers, $\rho=.32, p=.18$. Analyses of consistency yielded similar results.

\section{The effect of visual information}

\section{Dynamic movement reproduction accuracy.}

The 2 (Session: 1-2) x 2 (Visual Information: No visual information vs. Visual information) RM ANOVA analysis showed an effect of Session, $F(1,22)=4.57, p=.04, \eta_{p}^{2}=.17$, but no effect of Visual Information, $F<1$, and no interaction, $F<1$. Thus, visual information did not significantly influence DMR accuracy (Figure 7). The significant effect of Session suggests a potential learning effect (i.e., increased accuracy over sessions), as mean errors were significantly higher for Session $1(M=1.86 ; S E=0.14)$ than Session $2(M=1.64 ; S E=0.11)$.

Joint reposition accuracy.

A similar RM ANOVA showed an effect of Visual Information, $F(1,22)=7.42, p=.01, \eta_{p}^{2}$ $=.25$, and no effect of Session, $F<1$, or interaction, $F(1,22)=1.12, p=.30$. Including visual information increased accuracy (i.e., smaller mean errors; No visual information: $M=4.20$, $S E=0.30$; Visual information: $M=3.45, S E=0.30$; Figure 6).

\section{Study 2: Materials \& Methods}

The aim of the second study was to evaluate the reproducibility of findings regarding the association between both tasks and the influence of visual information using a larger, independent sample. Additionally, given the large sample, we considered the effect that task order might have on performance (first performing proprioceptive task with vision vs. first performing the task without vision). The apparatus, setting, procedure, and main outcome variables were identical to the first study, with the exception that this study was comprised of only one test session. In other words, participants performed both tasks only once, with the order of the tasks and conditions again randomized across participants.

\section{Participants}

A convenience sample of sixty-four healthy volunteers was recruited (recruitment sources and eligibility criteria identical to Study 1; sample size based on power calculations for anotherseparately preregistered - research question). Statistical analyses were run on the complete sample of $N=64$ (mean $(S D)$ age $=22.33$ (3.90), ranging from 18-37, 52 women). Participants received $€ 12.5$ in gift vouchers as compensation (part of a longer testing session).

\section{Data preparation and statistical analysis overview}


373

374

375

376

377

378

379

380

381

382

383

384

385

386

387

388

389

390

391

392

393

394

395

396

397

398

399

400

401

402

403

404

405

406

407

408

409

410

411

412

Data were checked and prepared as described above. DMR consistency, and JR accuracy and consistency data were not normally distributed. Analyses and inference criteria were also identical, with the exception that paired t-tests were used to test for the effect of visual information. Additional RM ANOVAs were used to explore order effects (visual information versus no visual information performed first).

\section{Study 2: Results}

Association between performance accuracy on the dynamic movement reproduction and joint reposition tasks (no visual information)

Spearman correlations suggest a weak positive relationship between DMR and JR accuracy (Table 1). Excluding outliers reduced the correlation further rendering it nonsignificant, $\rho=.22, p=.09$. Analyses of consistency yielded comparable results (excluding outliers: $\rho=.17, p=.19$ ).

\section{The effect of visual information}

Dynamic movement reproduction accuracy.

DMR accuracy differed significantly between conditions, $t(63)=-3.33, p<.005, d=.49$, showing higher errors when visual information was present $(M=1.81, S E=0.08)$ than when no visual information was present $(M=1.54, S E=0.06)$. These findings suggest poorer accuracy when vision was available. However, further exploratory analysis showed that condition order moderated the effect (Visual Information x Order: $F(1,62)=7.16, p<.01, \eta_{p}^{2}=.10$ ). More specifically, there was no significant difference between conditions when performing the task without visual information first $(p=.52)$, but there was a difference between conditions when performing the task with visual information first $(p<.001)$. In sum, visual information decreased accuracy, but only when the task started with visual information available.

\section{Joint reposition accuracy.}

JR accuracy differed significantly between conditions, $t(63)=3.49, p<.001, d=.59$, with lower errors when visual information was present $(M=2.82, S E=0.15)$ than when no visual information was present $(M=3.56, S E=0.17)$. Thus, participants showed poorer accuracy without visual information available. Adding the order of conditions as a factor in a RM ANOVA did not change the results (Visual Information x Order: $F<1$ ).

\section{Discussion}

We developed a new task to dynamically assess sensorimotor integration underlying kinesthetic proprioceptive function through measures of kinesthetic proprioceptive accuracy during upper limb movement, and evaluated test-retest reliability, association with a JR task, and the influence of visual input on task performance. Our first hypothesis, that the DMR task testretest reliability would be good to excellent was supported, as was the presence of weak associations between the DMR task and the JR task. Interestingly, allowing the use of vision during the DMR task was different than we hypothesized - vision only improved task 
413 performance for the JR task, not the DMR task. Importantly, our latter findings on vision and 414 task performance associations were largely reproducible in a larger independent sample, 415 although the effect of vision on DMR task performance was not.

416 An important implication of the present study is that our new DMR task evaluating 417 kinesthetic proprioceptive function exhibits sufficient test-retest reliability to support its use in 418 research and clinical settings. That is, DMR accuracy showed good-to-excellent reliability, and fair-to-good test-retest reliability was found for consistency (i.e., variation in error throughout

421

422

423

424

425

426

427

428

429

430

431

432

433

434

435

436

437

438

439

440

441

442

443

444

445

446

447

448

449

450

451

452 the task). These results are comparable to other studies evaluating test-retest reliability of tasks assessing proprioceptive function using robotic devices (e.g., Cappello et al., 2015; Rinderknecht et al., 2018; Rinderknecht, Popp, Lambercy, \& Gassert, 2016). Furthermore, this reliability was notably higher than that of the traditional JR task tested here. However, it is of interest that our findings of poor test-retest reliability for JR accuracy differed from that of Juul-Kristensen and colleagues' (2008b), who found fair-to-good reliability. There could be numerous reasons underlying these differences between our studies. While device precision appears comparable, it is possible that use of differing forearm repositioning angles (smaller angles in our task which may increase task ease, reducing between-subject variability and thus reliability), differing duration between test sessions ( 1 hour vs. 24 hours in present study, enhancing memory of the task and increased reliability in former work), participants' age range (18-57 years vs. 18-32 years in present study, reducing between-subject variability), and examiner experience (highly trained physiotherapists in past work, psychology student here) all contributed to differing findings. Together, our differing reliability findings for the JR task support past work showing that reliability varies widely (Clark et al., 2015; Elangovan et al., 2014). Regardless, it is important to highlight that potential memory and/or examiner effects did not influence the DMR measure to the same extent, thus highlighting the benefit of using a highly standardized protocol and a device allowing for precise measurement such as the HapticMaster.

The second implication of this study is that the DMR task measure of proprioceptive accuracy captures a unique aspect of proprioceptive function; that is, distinct from the JR task. Indeed, the association between DMR and JR accuracy was poor. Importantly, we replicated this finding in our second study using a larger sample, collected to remove any concerns about low statistical power in Study 1. While both tasks involve active reproductions involving the elbow joint of the dominant (right) arm which might suggest stronger association, in general, measures of proprioceptive function correlate weakly, as they assess different aspects of proprioception (De Jong, Kilbreath, Refshauge, \& Adams, 2005; Elangovan et al., 2014). In the DMR task, participants perform a specific movement pattern (i.e., a circle) and then reproduce this pattern. This is similar to the JR task where participants move their arm to a certain position and then reproduce it. That is, the DMR task involves remembering the position and size of the circle and using (combined) limb position sense to replicate that circular movement, as well as sensorimotor integration to ensure accurate performance of the intended action. The latter integration is likely underpinned via generation of a motor efferent copy, which is then compared to sensory input that has resulted as a consequence of the movement (Wolpert \& Ghahramani, 
453 2000; Wolpert et al., 1995). However, the purposeful complex movements during the DMR task 454 require higher levels of sensorimotor integration compared to the JR task. Furthermore, the error 455 measure in the DMR task is not only looking at an 'end position' but the accuracy of the entire 456 movement (i.e., dynamic assessment). Our findings suggest then that the DMR task mainly

457

458

459

460

461

462

463

464

465

466

467

468

469

470

471

472

473

474

475

476

477

478

479

480

481

482

483

484

485

486

487

488

489

490

491

492 captures sensorimotor integration processes underlying kinesthetic proprioceptive function, rather than joint position sense. Furthermore, it is of course, relevant to note that the DMR task involves the entire upper limb kinetic chain (shoulder, elbow and wrist joints) while the JR task only involves elbow movement, which may also contribute to poor associations. Together these results emphasize the importance of considering and appropriately assessing the proprioceptive feature of interest as well as the relevant joints for that condition. Overall, our findings support our hypothesis that the DMR task indeed captures a unique aspect of proprioceptive function compared to JR tasks.

Finally, our results suggest that the contribution of visual information to proprioceptive accuracy is complex. It appears dependent on the proprioceptive task, and for the DMR task, was opposite of what we expected. That is, while our first study found no difference between visual conditions when performing the DMR task, exploration of this in a larger sample showed lower accuracy when performing the task with visual information. Additionally, the condition order moderated this effect, as it was only present when participants started the task with visual information present. In contrast, JR results were consistent with expectations, indicating that visual information increased accuracy (Scheidt et al., 2005). These contradicting results of vision for the JR versus DMR task may be explained by task differences. For example, in the JR task, simple forearm positions are reproduced (i.e., allowing the use of visual reference points), while in the DMR task more complex movements (including movement of a robotic arm) using multiple joints are performed, potentially making reliance upon visual information a disadvantage. Further, order effects for the DMR task may be explained by the influence of visual information while learning a task. For example, if visual information is absent while learning a complex task, it is learned proprioceptively; the visual information that is available afterwards is then an adjunct to the proprioceptively learned task. The same may not occur if visual information is present while learning a complex task - it may provide less reliable information for a complex movement than the proprioceptive input. This emphasizes that the integration of visual and proprioceptive information is not straightforward (Sarlegna \& Sainburg, 2009; Scheidt et al., 2005), and supports that proprioceptive and visual information are weighted based on their reliability (van Beers, Sittig, \& van der Gon, 1999).

Some limitations of the current study should be outlined as well. First, analysis of the effect of visual information on the DMR task revealed the presence of a potential learning effect between sessions (i.e., higher accuracy in the second session), even though our initial analysis did not show this effect and test-retest reliability was in the good-to-excellent range. However, this may have led to an underestimation of test-retest reliability. Increasing practice or familiarization when using tasks that are more complex may be advisable. Second, we did not fully standardize movement kinematics of the upper limb during the DMR task, allowing some 
493 variation in use of different joints during movements (i.e., only movement of the sensor of the

494 robotic arm was recorded). When comparing accuracy within or between participants, this should

495 be standardized in order to prevent compensation for joint-specific deficits. Third, the sample

496 size of our first study may not have provided sufficient statistical power for the JR test-retest

497 analysis, although it was sufficient for the DMR measures. Fourth, since the average age of our

498 sample is relatively low, the present findings are limited in generalizability toward older adults.

499 Finally, as with all assessments of active proprioceptive function, factors other than

500 proprioception can influence the outcomes. For example, both the DMR and the JR methods are

501 less suitable for people with severe cognitive impairments since the tasks depend on working

502 memory (Han et al., 2016). Another factor is motor control: the precision of movement limits the

503 precision of the measured proprioceptive accuracy (Elangovan et al., 2014). However, motor

504 control and proprioception are closely related as both are integrated to perform movements

505 (Proske \& Gandevia, 2012).

506 The DMR task's highly automated (i.e., requiring limited operator input) and brief

507 (around $15 \mathrm{~min}$ ) protocol makes it straightforward to use in a clinical setting. Our results indicate

508 that a change of $.76 \mathrm{~cm}$ or more in DMR accuracy (i.e., the difference between two

509 measurements) is meaningful, and not merely due to measurement error (Beckerman et al.,

510 2001), highlighting its precision. It should be noted that the task presented here is not necessarily

511 limited to use of HapticMaster devices, as similar devices, commonly found in research and

512 rehabilitation settings (e.g., motor cortex retraining in stroke patients; Timmermans, Seelen,

513 Willmann, \& Kingma, 2009), could be used. Further, our DMR task using a robotic device,

514 while not the first, extends previous work in this area. For example, in contrast to our DMR task,

515 past active robotic tasks have depended on visual guidance (Dukelow et al., 2012), or used

516 mirror-matching (Kenzie et al., 2014). Additionally, Kitchen and Miall (2019) have used a

517 robotic device in older adults to evaluate arm-reaching movements, though their task involves

518 reaching a position along a straight line as quickly as possible, and thus does not require complex

519 sensorimotor integration. Rather, the DMR is the first to use vision occluded complex

520 movements, particularly emphasizing sensory guidance by using replication of a circular pattern

521 and not emphasizing speed, making it more appropriate to assess kinesthetic proprioceptive

522 function and the sensorimotor integration processes required for accurate arm movement pattern

523 reproduction.

524 The innovative aspect of our DMR task is that it dynamically assesses sensorimotor

525 integration processes of the entire upper limb kinetic chain, potentially allowing capture of more

526 complex kinesthetic proprioceptive deficits. In addition to the device's precise measurement, this

527 could help capture deficits in certain conditions that involve multi-joint impairment more

528 accurately, which is of importance in both research and practice (Röijezon et al., 2015;

529 Stasinopoulos, 2019). For example, lateral epicondylalgia (LE), or 'tennis elbow' is

530 characterized by symptoms of persistent pain and sensorimotor dysfunction, and people with LE

531 present with impaired proprioceptive function at the elbow (Juul-Kristensen et al., 2008a).

532 However, recent work has shown that sensorimotor dysfunction also occurs at the shoulder, the 
533 scapula, and the wrist (Alizadehkhaiyat, Fisher, Kemp, Vishwanathan, \& Frostick, 2007; Day, 534 Bush, Nitz, \& Uhl, 2015; Lucado, Kolber, Cheng, \& Echternach, 2012), and that it may be the 535 combination of impairment within this dynamic upper extremity kinetic chain that impedes 536 treatment (Lucado, Vincent, \& Day, 2019). However, reliable, valid proprioceptive tests to 537 evaluate the entirety of the upper limb kinetic chain are currently lacking, limiting detection of 538 impairment and provision of appropriate treatment (Stasinopoulos, 2019). Future research is 539 warranted to explore use of the DMR task in clinical populations such as LE, evaluating test540 retest reliability as well as exploring the predictive validity of the DMR measure for clinical 541 improvement via proprioceptive or movement retraining. Visual inspection of the reproduced

542 (versus target) movement pattern data may also prove useful in clinical application, as it may

543 allow identification of what aspect of proprioceptive function to target (see Supplementary

544 Material S1 for examples of impaired position sense, but intact sensorimotor integration and vice

545 versa). Further work exploring combination of the DMR task with movement capture systems to

546 explore differences in the way movement occurs during the task might provide interesting

547 insight.

548

549 Conclusions

550 In conclusion, the DMR task seems a promising new tool for reliably testing kinesthetic

551 proprioceptive function of the upper limb. It showed high test-retest reliability, and appears to

552 capture a unique aspect of proprioceptive function, as it dynamically evaluates sensorimotor

553 integration processes of the entire upper limb. This may make the DMR task particularly relevant

554 for certain clinical conditions with multiple-joint involvement. Additionally, this study shows

555 that the integration of visual and proprioceptive information is not straightforward, with vision of

556 arm movement beneficial during simple movements, but not when learning complex movements, 557 and supports the idea that proprioceptive and visual information are weighted based on their

558 task-specific reliability.

559

560 Acknowledgements

561 The authors wish to thank Jacco Ronner for programming the DMR task and Vanessa Lostaunau

562 Calero for assisting in data collection. 


\section{References}

564 Alizadehkhaiyat, O., Fisher, A. C., Kemp, G. J., Vishwanathan, K., \& Frostick, S. P. (2007).

565

566

567

568

569

570

571

572

573

574

575

576

577

578

579

580

581

582

583

584

585

586

587

588

589

590

591

592

593

594

595

596

597

598

599

600

601

602

603

604

605

606

607

608
Upper Limb Muscle Imbalance in Tennis Elbow: A Functional and Electromyographic Assessment Omid. Journal of Orthopaedic Research September, 25, 1651-1657. https://doi.org/10.1002/jor

Beckerman, H., Roebroeck, M. E., Lankhorst, G. J., Becher, J. G., Bezemer, P. D., \& Verbeek, A. L. M. (2001). Smallest real difference, a link between reproducibility and responsiveness. Quality of Life Research, 10(7), 571-578.

https://doi.org/10.1023/A:1013138911638

Cappello, L., Elangovan, N., Contu, S., Khosravani, S., Konczak, J., \& Masia, L. (2015). Robotaided assessment of wrist proprioception. Frontiers in Human Neuroscience, 9(APRIL), 18. https://doi.org/10.3389/fnhum.2015.00198

Chen, H. M., Chen, C., Hsueh, I. P., Huang, S. I., \& Hsieh, C. L. (2009). Test-Retest Reproducibility and Smallest Real With Stroke. Neurorehabilitation and Neural Repair, 23(17), 435-440.

Chowdhury, R. H., Glaser, J. I., \& Miller, L. E. (2020). Area 2 of primary somatosensory cortex encodes kinematics of the whole arm. ELife, 9, e48198. https://doi.org/10.1101/643205

Clark, N. C., Röijezon, U., \& Treleaven, J. (2015). Proprioception in musculoskeletal rehabilitation. Part 2: Clinical assessment and intervention. Manual Therapy, 20(3), 378387. https://doi.org/10.1016/j.math.2015.01.009

Davies, G. J., Krauschaar, D. J. R., Brinks, K. F., \& Jennings, J. (2006). Neuromuscular Static and Dynamic Stability of the Shoulder: The Key to Functional Performance. In Postsurgical Orthopedic Sports Rehabilitation: Knee \& Shoulder (pp. 133-155). https://doi.org/10.1016/B978-032302702-1.50010-7

Day, J. M., Bush, H., Nitz, A. J., \& Uhl, T. L. (2015). Scapular muscle performance in individuals with lateral epicondylalgia. Journal of Orthopaedic and Sports Physical Therapy, 45(5), 414-424. https://doi.org/10.2519/jospt.2015.5290

De Jong, A., Kilbreath, S. L., Refshauge, K. M., \& Adams, R. (2005). Performance in Different Proprioceptive Tests Does Not Correlate in Ankles With Recurrent Sprain, 2101-2105. https://doi.org/10.1016/j.apmr.2005.05.015

Dukelow, S. P., Herter, T. M., Bagg, S. D., \& Scott, S. H. (2012). The independence of deficits in position sense and visually guided reaching following stroke. Journal of NeuroEngineering and Rehabilitation, 9(1), 1-13. https://doi.org/10.1186/1743-0003-9-72

Elangovan, N., Herrmann, A., \& Konczak, J. (2014). Assessing Proprioceptive Function: Evaluating Joint Position Matching Methods Against Psychophysical Thresholds. Physical Therapy, 94(4), 553-561.

Fleiss, J. L. (1986). Design and analysis of clinical experiments. New York: John Wiley \& Sons. Goble, D. J. (2010). Proprioceptive Acuity Assessment Via Joint Position Matching: From Basic Science to General Practice. Physical Therapy, 90(8), 1176-1184. https://doi.org/10.2522/ptj.20090399

Han, J., Waddington, G., Adams, R., Anson, J., \& Liu, Y. (2016). Assessing proprioception: A critical review of methods. Journal of Sport and Health Science, 5, 80-90. https://doi.org/10.1016/j.jshs.2014.10.004

Helsen, W. F., Van Halewyck, F., Levin, O., Boisgontier, M. P., Lavrysen, A., \& Elliott, D. (2016). Manual aiming in healthy aging: does proprioceptive acuity make the difference? Age, 38(2). https://doi.org/10.1007/s11357-016-9908-z

Peer] reviewing PDF | (2020:11:55170:1:0:NEW 19 Feb 2021) 
609 Hillier, S., Immink, M., \& Thewlis, D. (2015). Assessing Proprioception : A Systematic Review

610

611

612

613

614

615

616

617

618

619

620

621

622

623

624

625

626

627

628

629

630

631

632

633

634

635

636

637

638

639

640

641

642

643

644

645

646

647

648

649

650

651

652

653

654 of Possibilities. Neurorehabilitation and Neural Repair, 29(10), 933 -949. https://doi.org/10.1177/1545968315573055

Juul-Kristensen, B., Lund, H., Hansen, K., Christensen, H., Danneskiold-Samsøe, B., \& Bliddal, H. (2008a). Poorer elbow proprioception in patients with lateral epicondylitis than in healthy controls: A cross-sectional study. Journal of Shoulder and Elbow Surgery, 17, 72 81. https://doi.org/10.1016/j.jse.2007.07.003

Juul-Kristensen, B., Lund, H., Hansen, K., Christensen, H., Danneskiold-Samsøe, B., \& Bliddal, H. (2008b). Test-retest reliability of joint position and kinesthetic sense in the elbow of healthy subjects. Physiotherapy Theory and Practice, 24(1), 65-72. https://doi.org/10.1080/09593980701378173

Kenzie, J. M., Semrau, J. A., Findlater, S. E., Herter, T. M., Hill, M. D., Scott, S. H., \& Dukelow, S. P. (2014). Anatomical correlates of proprioceptive impairments following acute stroke: A case series. Journal of the Neurological Sciences, 342(1-2), 52-61. https://doi.org/10.1016/j.jns.2014.04.025

Kenzie, J. M., Semrau, J. A., Hill, M. D., Scott, S. H., \& Dukelow, S. P. (2017). A composite robotic-based measure of upper limb proprioception. Journal of NeuroEngineering and Rehabilitation, 14(1), 1-12. https://doi.org/10.1186/s12984-017-0329-8

Kitchen, N. M., \& Miall, R. C. (2019). Proprioceptive deficits in inactive older adults are not reflected in fast targeted reaching movements. Experimental Brain Research, 237(2), 531545. https://doi.org/10.1007/s00221-018-5440-y

Laraway, S., Snycerski, S., Pradhan, S., \& Huitema, B. E. (2019). An Overview of Scientific Reproducibility: Consideration of Relevant Issues for Behavior Science/Analysis. Perspectives on Behavior Science, 42(1), 33-57. https://doi.org/10.1007/s40614-01900193-3

Lucado, A. M., Kolber, M. J., Cheng, M. S., \& Echternach, J. L. (2012). Upper extremity strength characteristics in female recreational tennis players with and without lateral epicondylalgia. Journal of Orthopaedic and Sports Physical Therapy, 42(12), 1025-1031. https://doi.org/10.2519/jospt.2012.4095

Lucado, A. M., Vincent, J., \& Day, J. M. (2019). Response letter to the role of proprioception of lateral elbow tendinopathy. Journal of Hand Therapy, 32(1), e4-e5. https://doi.org/10.1016/j.jht.2018.04.002

Maggioni, S., Melendez-Calderon, A., Van Asseldonk, E., Klamroth-Marganska, V., Lünenburger, L., Riener, R., \& Van Der Kooij, H. (2016). Robot-aided assessment of lower extremity functions: A review. Journal of NeuroEngineering and Rehabilitation, 13(1), 125. https://doi.org/10.1186/s12984-016-0180-3

McGraw, K. O., \& Wong, S. P. (1996). Forming Inferences about Some Intraclass Correlation Coefficients. Psychological Methods, 1(1), 30-46. https://doi.org/10.1037/1082989X.1.1.30

Proske, U., \& Gandevia, S. C. (2012). The proprioceptive senses: their roles in signaling body shape, body position and movement, and muscle force. PhysiolRev, 92, 1651-1697. https://doi.org/10.1152/physrev.00048.2011

Rinderknecht, M. D., Lambercy, O., Raible, V., Büsching, I., Sehle, A., Liepert, J., \& Gassert, R. (2018). Reliability, validity, and clinical feasibility of a rapid and objective assessment of post-stroke deficits in hand proprioception. Journal of NeuroEngineering and Rehabilitation, 15(1), 1-15. https://doi.org/10.1186/s12984-018-0387-6 
655

656

657

658

659

660

661

662

663

664

665

666

667

668

669

670

671

672

673

674

675

676

677

678

679

680

681

682

683

684

685

686

687

688

689

690

691

692
Rinderknecht, M. D., Popp, W. L., Lambercy, O., \& Gassert, R. (2016). Reliable and rapid robotic assessment of wrist proprioception using a gauge position matching paradigm. Frontiers in Human Neuroscience, 10(June), 1-12. https://doi.org/10.3389/fnhum.2016.00316

Röijezon, U., Clark, N. C., \& Treleaven, J. (2015). Proprioception in musculoskeletal rehabilitation: Part 1: Basic science and principles of assessment and clinical interventions. Manual Therapy, 20(3), 368-377. https://doi.org/10.1016/j.math.2015.01.008

Saradjian, A. H. (2015). Sensory modulation of movement, posture and locomotion. Clinical Neurophysiology, 45, 255-267. https://doi.org/10.1016/j.neucli.2015.09.004

Sarlegna, F. R., \& Sainburg, R. L. (2009). Progress in Motor Control. In D. Sternad (Ed.), Progress in motor control (pp. 317-335). Boston, MA: Springer. https://doi.org/10.1007/978-0-387-77064-2

Scheidt, R. A., Conditt, M. A., Secco, E. L., \& Mussa-Ivaldi, F. A. (2005). Interaction of visual and proprioceptive feedback during adaptation of human reaching movements. Journal of Neurophysiology, 93(6), 3200-3213. https://doi.org/10.1152/jn.00947.2004

Shrout, P. E., \& Fleiss, J. L. (1979). Intraclass correlations: uses in assessing rater reliability. Psychological Bulletin, 86(2), 420-428. https://doi.org/10.1037/0033-2909.86.2.420

Stasinopoulos, D. (2019). The role of proprioception in the management of lateral elbow tendinopathy. Journal of Hand Therapy, 32(1), e2-e3. https://doi.org/10.1016/j.jht.2018.02.010

Timmermans, A. A., Seelen, H. A., Willmann, R. D., \& Kingma, H. (2009). Technology-assisted training of arm-hand skills in stroke: Concepts on reacquisition of motor control and therapist guidelines for rehabilitation technology design. Journal of NeuroEngineering and Rehabilitation, 6(1). https://doi.org/10.1186/1743-0003-6-1

van Beers, R. J., Sittig, A. C., \& van der Gon, J. J. D. (1999). Integration of Proprioceptive and Visual Position-Information: An Experimentally Supported Model. Journal of Neurophysiology, 81(3), 1355-1364.

Wolpert, D. M., \& Ghahramani, Z. (2000). Computational principles of motor neuroscience. Nature Neuroscience, 3, 1212-1217.

Wolpert, D. M., Ghahramani, Z., Jordan, M. I., Wolpert, D. M., Ghahramani, Z., \& Jordan, M. (1995). An Internal Model for Sensorimotor Integration Published by : American Association for the Advancement of Science Stable URL : https://www.jstor.org/stable/2889276 American Association for the Advancement of Science is collaborating with JSTOR to digitize. Science, 269, 1880-1882.

Wolpert, D. M., Goodbody, S. J., \& Husain, M. (1998). Maintaining internal representations: The role of the human superior parietal lobe. Nature Neuroscience, 1(6), 529-533. https://doi.org/10.1038/2245 


\section{Figure 1}

Exemplary flowchart of a single test session

Order of tasks and conditions (with or without visual information; indicated by emoji) was randomized across participants. In this example Dynamic Movement Reproduction (DMR) task is first, and Joint Reposition (JR) task is second. For DMR task, movement direction (clockwise or counterclockwise; indicated by arrows) was counterbalanced across conditions, and practice movements were squares, while test movements were circles, to minimize training effects.

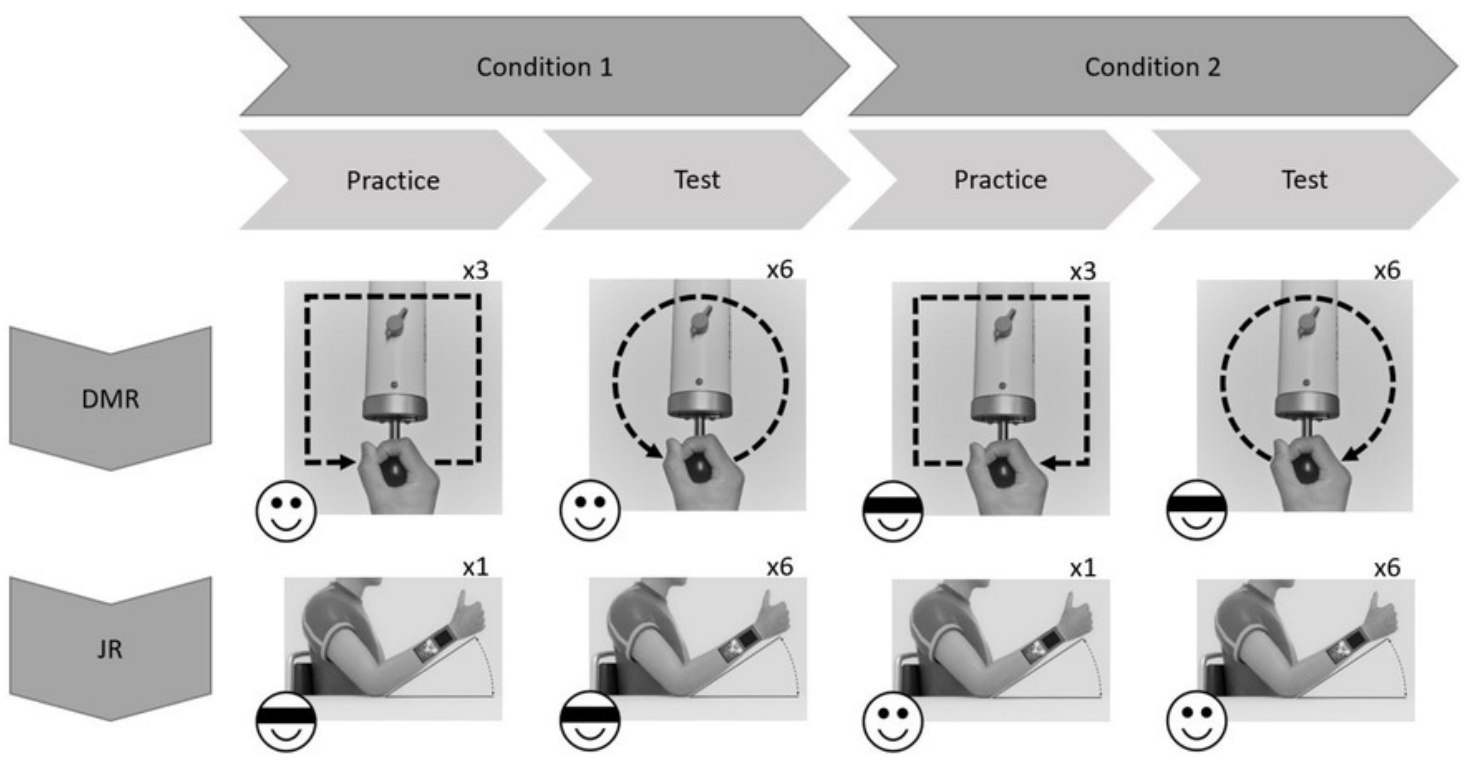




\section{Figure 2}

Experimental setup and trial flow of Dynamic Movement Reproduction task.

Note that during target movements the robotic arm haptically delineated the trajectory, while during movement reproduction there was no guidance. On the right side of the participant, a partition (not displayed here) separated the experimenter and participant to prevent potential distractions during performance of the task. 


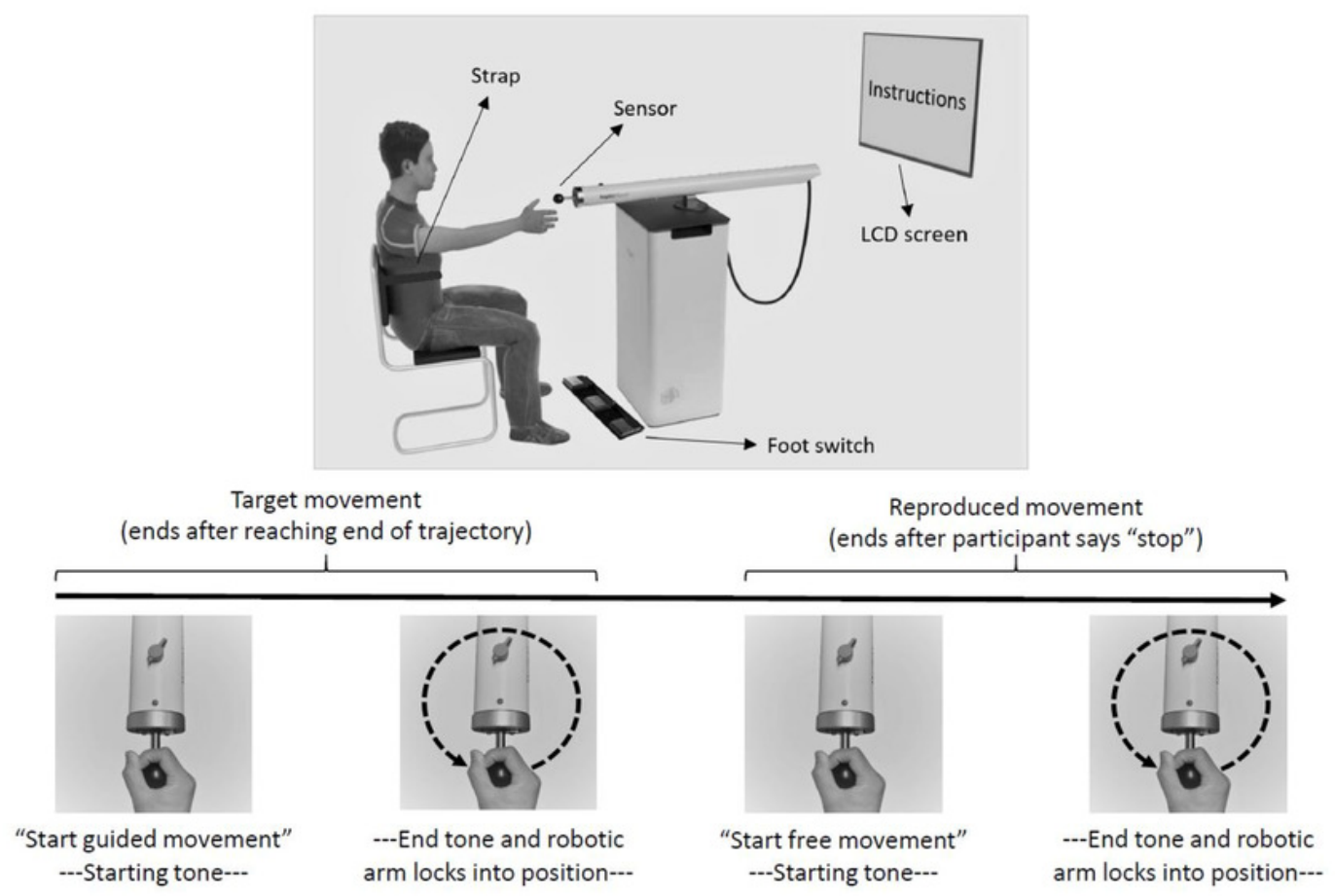


Figure 3

Trial flowchart with experimenter's verbal instructions during Joint Reposition tas 


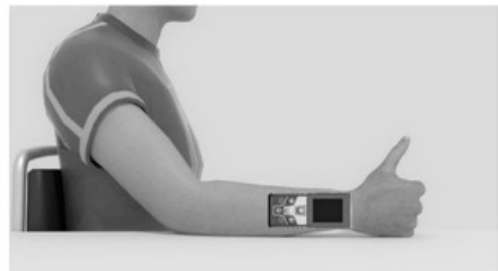

"Slowly, flex your arm, until I say stop."

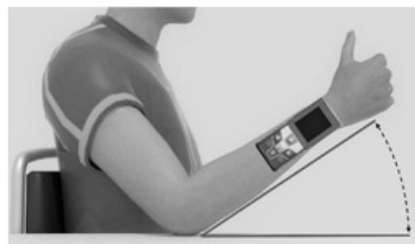

"Stop, hold and remember this position" --Experimenter registers angle--

"Return your arm to the table and keep it there"

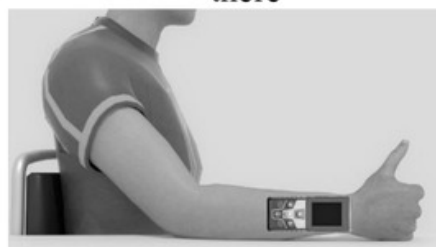

"Slowly flex your arm again to the same position as before and say, "Stop" when you think you reached it;"

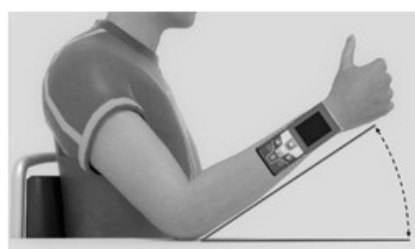

--Experimenter registers angle after participants says "Stop"--- 
Figure 4

Visualization of raw data from a single test trial of the Dynamic Movement Reproduction task

Both the target (red line) and reproduced (green line) movement pattern are visualized. Note that the dashed arrow represents the target radius, while the solid arrow represents the reproduced radius, which is calculated for each coordinate logged by the HapticMaster. 


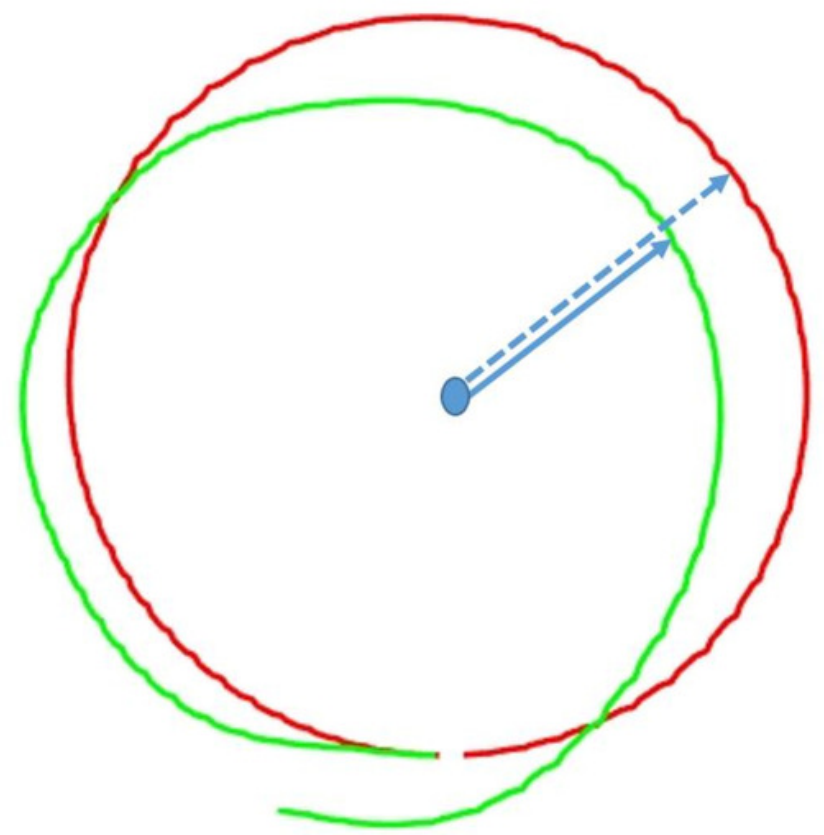




\section{Figure 5}

Plots of test-retest data of Dynamic Movement Reproduction accuracy (A) and consistency (B) with no visual information

The difference between Sessions 1 and 2 is plotted against the mean of both sessions. The mean difference between sessions is presented as a horizontal line (middle line), and the upper and lower lines represent the $95 \%$ upper and lower limits of these differences. Note that sufficient test-retest reliability corresponds with differences between sessions (y-axis) being closer to zero (i.e., roughly the same accuracy and consistency in both sessions), and variation in means between sessions ( $\mathrm{x}$-direction) being larger than variation in differences between sessions (y-direction; i.e., larger between-subjects variation than withinsubjects variation). 
A

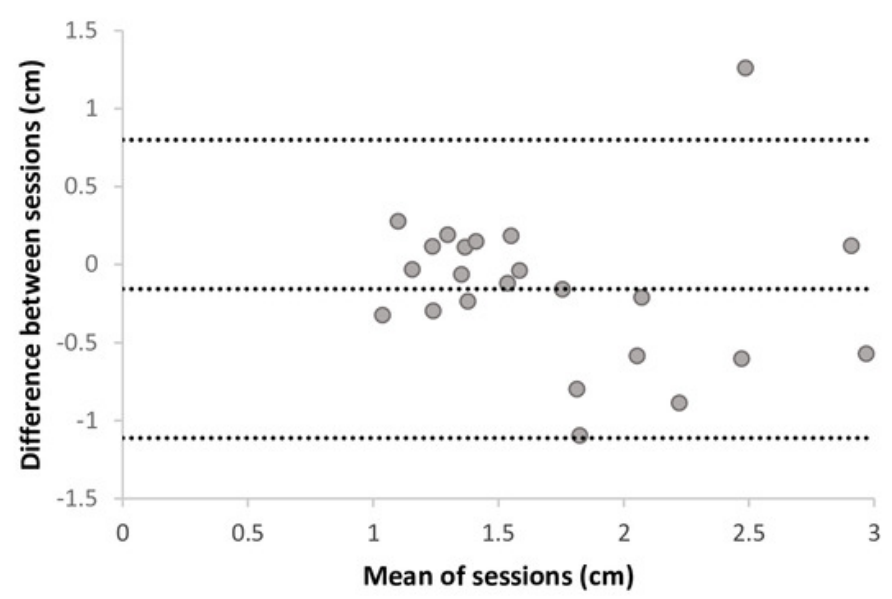

B

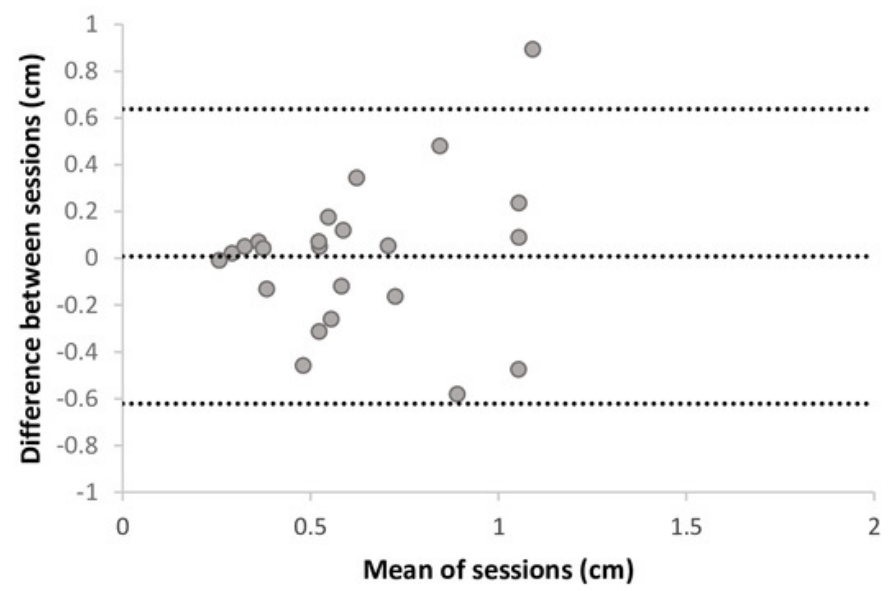




\section{Figure 6}

Plots of test-retest data of Joint Reposition accuracy (A) and consistency (B) with no visual information

The difference between Sessions 1 and 2 is plotted against the mean of sessions. The mean difference between sessions is presented as a horizontal line (middle line), and the upper and lower lines represent the $95 \%$ upper and lower limits of these differences. Note that sufficient test-retest reliability corresponds with differences between sessions ( $y$-axis) being closer to zero (i.e., roughly the same accuracy and consistency in both sessions), and variation in means between sessions ( $x$-direction) being larger than variation in differences between sessions (y-direction; i.e., larger between-subjects variation than within-subjects variation). 
A

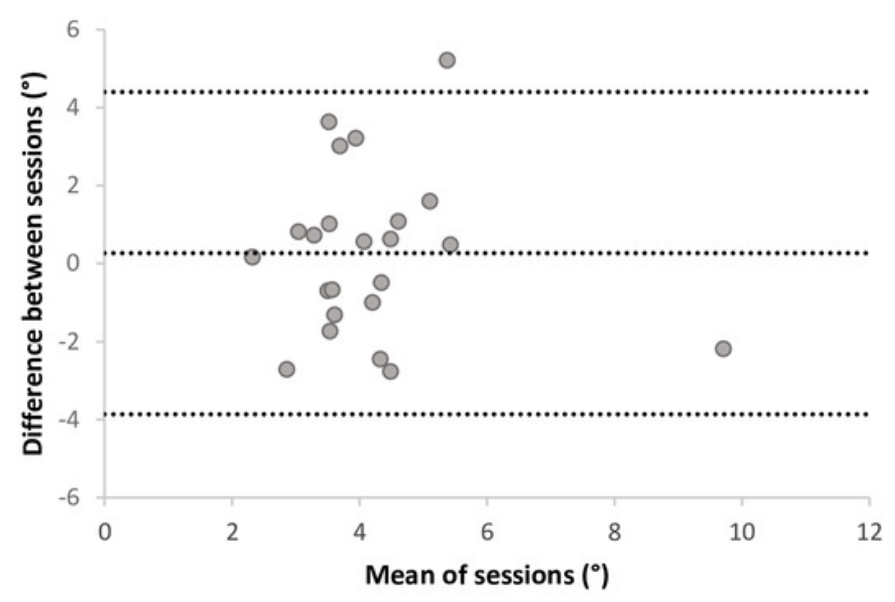

B

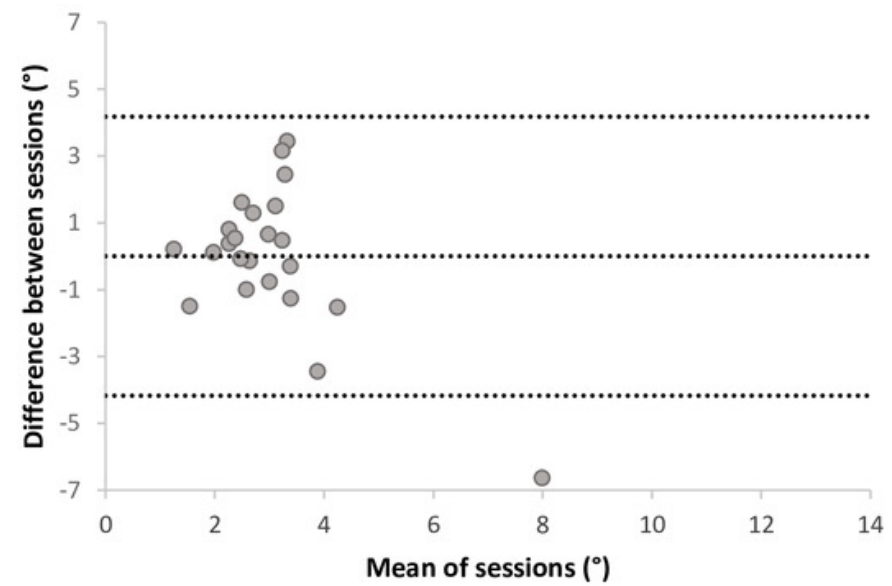


Figure 7

Joint Reposition (JR) and Dynamic Movement Reproduction (DMR) accuracy with and without visual information during studies 1 (test and retest) and 2

Higher values correspond with poorer accuracy. Error bars represent standard errors. 

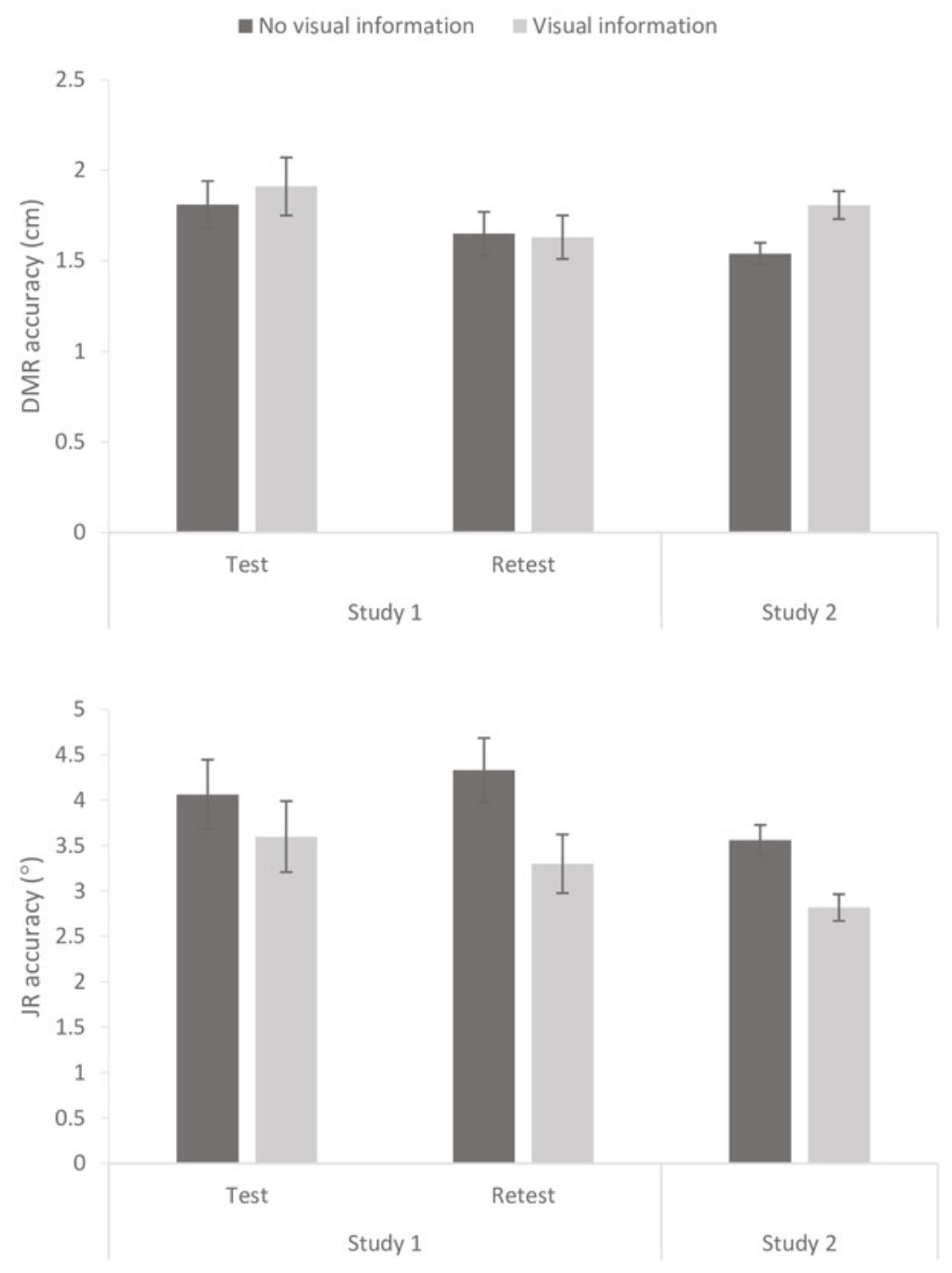


\section{Table $\mathbf{1}$ (on next page)}

Spearman correlations ( $\rho$ ) between Dynamic Movement Reproduction (DMR) and Joint Reposition (JR) accuracies, and consistencies (no visual information)

*Correlation is significant at the .05 level (2-tailed). No corrections for multiple testing. 


\begin{tabular}{|c|c|c|c|c|c|}
\hline \multirow[t]{2}{*}{$\rho$ (p-value) } & & \multicolumn{2}{|c|}{ JR accuracy } & \multicolumn{2}{|c|}{ JR consistency } \\
\hline & & Test & Retest & Test & Retest \\
\hline \multicolumn{6}{|l|}{ Study 1} \\
\hline \multirow[t]{2}{*}{ DMR accuracy } & Test & $.08(.72)$ & $.39(.07)$ & & \\
\hline & Retest & $.05(.81)$ & $.52 *(.01)$ & & \\
\hline \multirow[t]{2}{*}{ DMR consistency } & Test & & & $.06(.77)$ & $.54 *(.01)$ \\
\hline & Retest & & & $.02(.93)$ & $.27(.22)$ \\
\hline \multicolumn{6}{|l|}{ Study 2} \\
\hline DMR accuracy & Test & $.25 *(.05)$ & & & \\
\hline DMR consistency & Test & & & $.22(.08)$ & \\
\hline
\end{tabular}

1 Note. *Correlation is significant at the .05 level (2-tailed). No corrections for multiple testing. 